\title{
Finite Element Simulation of Moving Targets in Radio Therapy
}

\author{
Pan Li, Gregor Remmert, Jürgen Biederer, Rolf Bendl \\ Medical Physics, German Cancer Research Center, 69120 Heidelberg \\ Email: pan.li@dkfz.de
}

\begin{abstract}
Among other options, radiotherapy plays an increasing role for the treatment of lung cancer. This has raised the need for strategies to compensate for respiratory motion of the target. Sophisticated techniques such as tracking of the tumour position have become feasible. However, calculations for adequate dose delivery to moving targets with maximum preservation of healthy tissue require understanding the principle of soft tissue deformation In this paper, we introduce a new approach using non-linear finite element method to simulate lung tissue displacement during respiration. The geometric non-linearity and the material non-linearity were applied. The respiration movement was regarded as steady gradual process with equal velocity, and was simulated statically. Boundary condition was set on lung's surface, and the deformation of whole volume could be calculated. Simulation of diaphragm breathing was compared with $4 \mathrm{DCT}$ of ventilated porcine lung inside a chest phantom. The average difference of internal point movement was 3 $\mathrm{mm}$ vertically. Our vision is to integrate the concept of FEM simulation of respiratory motion into motion adapted radiotherapy planning.
\end{abstract}

\section{Introduction}

Among other options, radiotherapy plays an increasing role for its treatment. The key strategy of radiotherapy is to maximize local dose at the target, while at the same time minimizing damage to adjacent normal tissue. The easiest approach to account for target movement with respiration is to define larger planning target which covers the malignant lesion at any point of its course during the respiratory cycle. Meanwhile, techniques for gated irradiation at certain positions or for tracking the tumour during the respiratory cycle should principally allow for smaller planning target volumes. To understand the principle reason of soft tissue deformation due to external force has become a key for defining planning target volumes for motion-adapted radiation. Over the last three decades the biomechanism of soft tissue has been thoroughly investigated. Based on theoretical knowledge and recent technique of CT, MRI, biomedical simulation has been widely used in surgical planning system, functional assessment of organ (e.g. heart) or molecular biological simulation. However, less work has been done to simulate lung movement. The objective of this work is to introduce a new approach of finite element simulation to describe lung tissue deformation and to integrate it in radiotherapy planning. 


\section{State of the art and new contribution}

Segars [1] describe structures inside the torso with a set of spline-based geometrical primitives. But he did not give any physical law for that model. Wu [2] implemented an adaptive FEM model for soft tissue. But no further work in lung simulation was given. In our FEM simulation we will study the internal deformation of lung.

\section{Methods}

Normal lung can be considered as homogeneous, isotropic, non-linear compressible object. Its non-linearity can be divided as geometric non-linearity (non-linear strain) and non-linear material property. Since no in vivo material law for lung was published so far, we employed simpler hyperelastic law. Another material which is more reasonable for soft tissue, was introduced by Veronda and Westermann [3]. This was also integrated in our work.

\subsection{D volume mesh}

A FEM pre-processor was developed to generate 3D tetrahedron volume mesh and assign material properties. The $3 \mathrm{D}$ mesh was achieved firstly by initiating volume of interest (VOI) with volume grower segmentation algorithm, retrieving surface mesh with marching cube, and decomposing volume in tetrahedron elements with Netgen [4]. Assigning material properties was done in a graphic interactive process. According to its neighbourhood, each element on the boundary was labelled with one material index which represents its neighbour organ, e.g. elements which share boundary with diaphragm, were set with diaphragm index. All internal elements have the same index. Usually, meshing program, especially Delauny algorithm, couldn't assure mesh quality. Since FEM simulation works with volume mesh data, it has high demand for the mesh quality. We implemented a so-called steepest descendent approach to improve mesh quality. With $V, L$ referring to volume and length of edges and $C$ is the normalizing factor, the measure referring to tetrahedron quality was defined as

$$
Q=C \frac{V}{L^{3}}
$$

\subsection{Finite element method}

Here, we briefly introduce the problem which need to be solved. $\Omega 0$ is the undeformed state of the elastic object. The principle of virtual work in the total lagrangian formulation is given by

$$
\int_{\Omega 0}{ }^{t+\Delta t} S \delta_{0}^{t+\Delta t} \varepsilon d^{0} V={ }^{t+\Delta t} R
$$


where ${ }_{0}^{t+\Delta t} S$ is second Piolar Kirchhoff stress tensor, ${ }_{0}^{t+\Delta t} \varepsilon$ is lagrangian strain tensor, operator $\delta$ indicates small virtual displacement. ${ }^{t+\Delta t} R$ denotes to external virtual work. The small 0 means each item is related to referential configuration. Based of its non-linear property, equation (2) must be solved in an incremental way. $t$ refers to any arbitrary time point, $\Delta t$ implies time increment. The incremental decomposition of the non linear strain representing geometry non-linearity turned out to be an incremental part and a part related to the last time step. The incremental part can be further decomposed as linear and non-linear components. After linearizing the higher-order term in the non-linear component, we obtain the final equation of motion with ${ }_{0} C$ denoting the material elastic tensor

$$
\int_{\Omega 0}{ }_{0} C_{0} e \delta_{0} e d^{0} V+\int_{\Omega 0}{ }_{0}^{t} S \delta_{0} \eta d^{0} V+\int_{\Omega 0}{ }_{0}^{t} S \delta_{0} e d^{0} V={ }^{t+\Delta t} R
$$

Biomedical material always shows non linear, non elastic properties. Wu included both Mooney-Rivlin and Neo-Hookean material law which depict linear relation of strain energy and Cauchy deformation tensor. Veronda and Westermann introduced another material law which can express the feature of soft tissue through a exponential term. We included both Neo-Hookean and Veronda Westmann material law.

\section{Results}

We tested our finite element simulator with ventilated ex-vivo system. The phantom experiment used a diaphragmatic pump for the exactly reproducible ventilation of animal lung explants inside a dedicated porcine chest phantom [5]. Before CT scanning 8 markers were injected into the left and right lung, respectively 4 markers in each side. 4DCT lung image data was segmented to generate mesh in the following steps. Then the segmented region was labelled with a gray value, and the rest of the image cube was set with zero. In the next step the labelled image cube was transferred to a surface retrieving module. A water tight iso-surface triangle mesh was retrieved by using marching cube algorithm. Finally, the volume surrounded by the triangle surface mesh was decomposed into tetrahedron 3D mesh with software Netgen. To verify the conformity of our 3D lung model to the real lung, we compared it with the original $3 \mathrm{D}$ surface image reconstructed by a in house developed software.

Figure 1a shows segmentation of the right lung in one slice. Figure 1a indicates, there is no great mismatch between the real lung surface image and the 3D model. Figure 2a illustrates a kind of tetrahedron element with bad quality which is called sliver. Figure $2 \mathrm{~b}$ gives the result of improved quality after 10 iterations. After obtaining 3D volume mesh, material properties were set to surface elements regarding their direct neighbours. Figure 3a depicts the boundary conditions.

First, we tried pseudo-simulation of diaphragm inspiration. Normally, it's difficult to measure the external surface force during the lung ex- or inspiration. Therefore, we defined surface nodes displacement for diaphragm instead 
Fig. 1. Lung contour (a) and model (b): original (red), model (green)

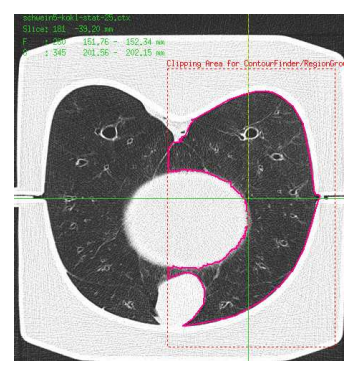

(a)

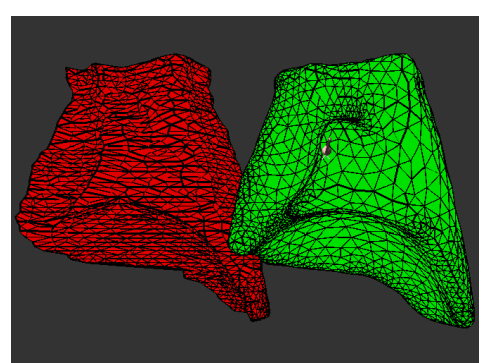

(b)

Fig. 2. Tetrahedon optimization: top tetrahedron with bad volume edge length behaviour (a) and optimized tetrahedon (b)

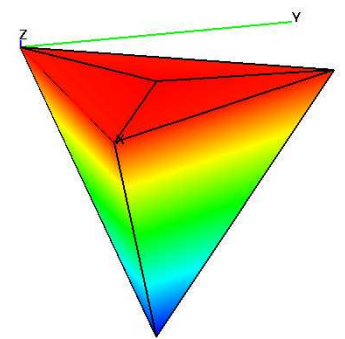

(a)

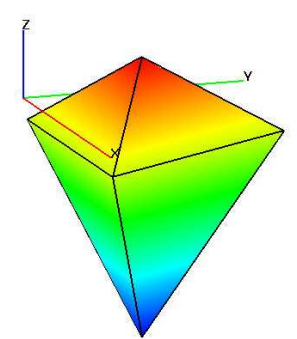

(b)

of setting external force. The whole body deformation was driven by its continuous surface nodes displacement. To simulate diaphragm inspiration, we drove down all surface nodes on diaphragm vertically with different increments. The sliding of other surface nodes along the pleural membrane was simulated by the displacement allowed only parallel to their surface planes.

Figure 3b illustrates the whole process of FEM simulation. Figure 3c demonstrates the deformed state. In Table 1 the result (in mm) was compared with 4DCT data.

The simulation was done in 250 steps. The computation of each incremental step took around 6 seconds for 5190 elements, which corresponds to 3 Newton iterations.

\section{Discussion}

The pseudo diaphragm inspiration was simulated by vertically moving surface nodes on diaphragm. However, in real anatomy the situation is far more complex. The pleural sliding between lung bottom and diaphragm must be considered. And no node moves only in one direction. There are two approaches to improve our simulation. First, inspiration can be considered as driven by external force. 
Fig. 3. Boundary conditions (a), incremental solution (b) and deformed state (c) with diaphragm (green), lung (red), upper part (white) and small elements (yellow)

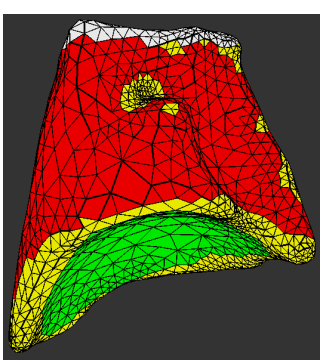

(a)

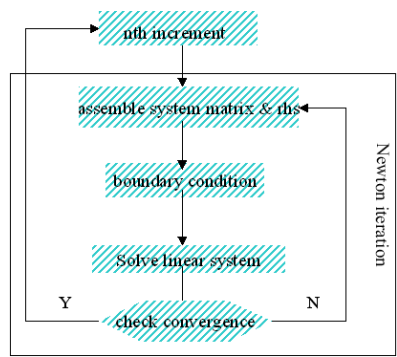

(b)

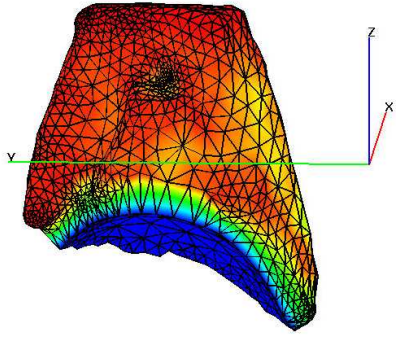

(c)

Table 1. Results: number of implants $(N)$, displacement $(x, y, z)$

\begin{tabular}{lllllll}
\hline \multirow{2}{*}{$\mathrm{N}$} & \multicolumn{2}{c}{ displacement in CT $[\mathrm{mm}]$} & \multicolumn{3}{c}{ Result of FEM simulation [mm] } \\
& $x$ & $y$ & $z$ & $x$ & $y$ & $z$ \\
\hline 1 & 1.170 & 0.000 & -22.4 & 0.653 & 0.043 & -19.6 \\
2 & -1.170 & 2.350 & -18.4 & 0.677 & -0.110 & -20.0 \\
3 & 0.000 & -0.560 & -13.6 & -1.020 & -1.040 & -10.3 \\
4 & -2.340 & 1.760 & -20.6 & 0.176 & 1.337 & -17.0 \\
\hline
\end{tabular}

In this case we have to calculate surface traction and its contribution to each surface node. Second, we can include diaphragm in our model and deal with frictionless contact problem between lung and diaphragm. Soft tissue always shows viscoelastic property. Lung should not be modelled only with hyperelastic material law. New material law must be studied. These will be done in our future work. Noted in the introduction, our goal is to apply the new FEM simulation to predict invisible tumor movement. Since techniques for the tracking of respiratory motion with external markers are available, it remains a challenge to use this signal for the calculation of invisible internal tumor movement with FEM simulation. Our vision is to generate a virtual 3D model for motion adapted radiotherapy planning based on the presented technology.

\section{References}

1. Segars WP, et al. Modeling respiratory mechanics in the MCAT and spline-based MCAT phantoms. IEEE Trans Nucl Sci 2001;48(1):89-97.

2. $\mathrm{Wu} \mathrm{X}$, et al. Adaptive nonlinear finite elements for deformable body simulation using dynamic progressive meshes. Procs Eurographics 2001;20(3):349-358.

3. Veronda DR, Westmann RA. Mechanical characterization of skin-finite deformations. J Biomech 1970;3:111-124.

4. Schöberl J. Netgen; 2007. http://www.hpfem.jku.at/netgen/.

5. Biederer J, et al. Reproducible simulation of respiratory motion in porcine lung explants. Fortschr Röntgenstr 2006;178:1067-72. 